\title{
Druckentlastung (Unloading) im Kniegelenk in Kombination mit knorpelchirurgischen Eingriffen
}

\author{
Clinical Relevance of Unloading in Cartilage Therapy of the Knee - \\ Shoe Insoles, Knee Braces or Additional Operative Procedure?
}

Autoren

Institute
T. M. Kraus ${ }^{1,2}$, A. B. Imhoff ${ }^{2}$, A. Ateschrang ${ }^{1}$, U. Stöckle ${ }^{1}$, S. Schröter ${ }^{1}$

${ }^{1}$ BG Unfallklinik, Eberhard-Karls-Universität Tübingen

${ }^{2}$ Abteilung für Sportorthopädie, Klinikum rechts der Isar der TU München
Schlüsselwörter

- Entlastung

- Kniegelenksorthese

- Umstellungsosteotomie

- HTO

- Knorpelschaden

Key words

- unloading

- knee brace

- unloading osteotomy

- high tibial osteotomy

- cartilage lesion
Bibliografie

DOI http://dx.doi.org/ 10.1055/s-0034-1396228

Z Orthop Unfall 2015; 153:

75-79 @ Georg Thieme

Verlag KG Stuttgart · New York .

ISSN 1864-6697

\section{Korrespondenzadresse}

PD Dr. Atesch Ateschrang

Sektionsleiter Sporttrauma-

tologie und arthroskopische

Chirurgie

BG Unfallklinik

Eberhard-Karls-Universität

Tübingen

Schnarrenbergstraße 95

72076 Tübingen

Tel.: $07071 / 606-1166$

Fax: 07071/606-1186

aateschrang@

bgu-tuebingen.de

\section{License terms}

\section{$(\circledast) \Theta \circledast$}

\section{Zusammenfassung}

$\nabla$

Bei fokalen Knorpelschäden des Kniegelenks spielt neben einem knorpelchirurgischen Vorgehen die Entlastung des betroffenen Gelenkbereichs eine entscheidende Rolle. Die Entlastung eines geschädigten Gelenkbereichs kann durch Körpergewichtsreduktion, varisierende oder valgisierende Orthesen, durch operative Eingriffe wie arthroskopische Kompartmentententlastung durch Bandstichelung des Ligamentum obliquium posterior oder durch Umstellungsosteotomien erreicht werden. Das knorpelchirurgische und das entlastende Verfahren sollten individuell auf die vorliegende Pathologie und den einzelnen Patienten abgestimmt sein. Die Druckentlastung im Kniegelenk kann langfristig nur durch operative Verfahren erfolgen. Schuhranderhöhungen bzw. Einlagen erreichen nur eine begrenzte Entlastung. Kniegelenksorthesen können dieses Ziel erreichen, haben bei gewünschtem Effekt jedoch eine geringe Patientenakzeptanz.

\section{Einleitung $\nabla$}

Neben der direkten Knorpeltherapie wie Mikrofrakturierung (MFX) [1], Knorpel-Knochen-Transplantation (OATS) [2] oder der autologen Chondrozytentransplantation (ACT) [3] bei isolierten Knorpelschäden ist die Druckentlastung oder auch „unloading“ des betroffenen Gelenkabschnitts der zentrale Pfeiler der gelenkerhaltenden Therapie des Kniegelenks. Nach ACT in Kombination mit

\section{Abstract \\ $\nabla$}

Restoration of a neutral biomechanical environment and reduction of overload is an important factor contributing to the success of any cartilage repair procedure. Reduction of overload can by achieved by so called unloading procedures in order to reduce intraarticular pressure from the repair zone. Unloading can be achieved via loss of weight, wedged shoe insoles, knee braces or via operations such as osteotomies around the knee joint. The cartilage therapy and the concomitant unloading procedure should be adapted to the individual pathology and realistic aims of the patient. Wedged insoles and braces are the least invasive treatment methods. In comparison, however, beneficial effects of braces outline those of laterally wedged heels. Nevertheless long-term compliance with insoles and braces is poor. Concerning braces either because the positive effects of the braces are too small or because the adverse effects are too large. Unloading in the long run may only be achieved through operative procedures. When an osteotomy seems to be too invasive the arthroscopic release of the posterior oblique ligament might be an option. Patients with an intact contralateral chondral status, medium to slight malalignment who want to remain at high activity levels, remain good candidates for unloading osteotomies.

einer Beinachskorrektur mittels Open-WedgeHTO (HTO: high tibial osteotomy) zeigte sich eine geringere Versagensrate im Vergleich zur isolierten ACT [4].

In biomechanischen Untersuchungen konnten Agneskirchner et al. die intraartikuläre Druckverteilung in Kniegelenken mit verschiedenen Achsdeviationen bestimmen [5]. Bei gerader Beinachse fand sich im medialen Kompartiment ein leicht vermehrter intraartikulärer Druck im Ver- 


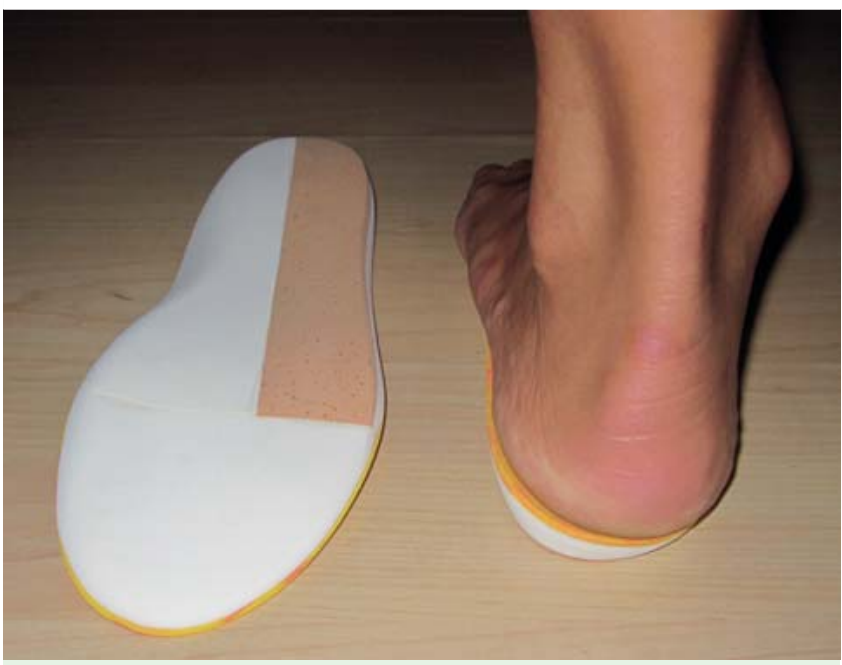

Abb. 1 Einlegesohle mit lateraler Randerhöhung.

gleich zum lateralen Kompartiment. Das Kräfte- und Druckverhältnis zwischen dem medialen und lateralen Kompartiment ist entsprechend der Studien von Agneskirchner et al. [5] und Mina et al. [6] zwischen einem mTFA (mechanischer tibiofemoraler Winkel) $0^{\circ}$ und $4^{\circ}$ valgus ausgeglichen. Bei zunehmend varischer Beinachse steigert sich der Druck medial um ca. $5 \%$ pro $1^{\circ}$ Achsabweichung [7]. Ebenso spielt die Bandspannung des Innenbands eine erhebliche Rolle. Agneskirchner et al. konnten zeigen, dass nach Open-Wedge-HTO eine ausreichende Entlastung erst nach einem Release von 50\% des Innenbands erreicht werden konnte [5].

Intraartikuläre Druckspitzen sind vor allem an den Rändern von chondralen Defektzonen nachweisbar. Die Drucksteigerungen um Knorpeldefekte herum waren bis zu einer Größe von $10 \mathrm{~mm}$ größenunabhängig. Ab einer Defektgröße von $10 \mathrm{~mm}$ Durchmesser oder einer Fläche von $0,79 \mathrm{~mm}^{2}$ hingegen wurden am Rand der Defekte größenabhängige Drucksteigerungen festgestellt [8]. Guettler et al. konnten selbst Drucksteigerungen in einem Radius von 2,2 mm um einen Knorpeldefekt nachweisen [8]. Ein intakter Meniskus kann anfallende Druckspitzen allenfalls um kleinere Defektgrößen bis $10 \mathrm{~mm}$ Durchmesser [9] absorbieren. Diesen Drucksteigerungen um größere Knorpeldefekte herum wird eine weitere knorpelschädigende Wirkung zugeschrieben [9]. Ergänzend hierzu fand die Arbeitsgruppe um Cicuttini et al. heraus, dass vorgeschädigter Knorpel eine schnellere Degeneration erfährt als gesunder Knorpel [10]. Brouwer et al. [11] konnten zeigen, dass alleine die Varusdeformität ein 2-fach höheres Risiko für die Entstehung einer Gonarthrose darstellt. Besteht zudem ein Meniskusschaden wird der Effekt des Überdrucks noch verstärkt [9]. Aus diesen beschriebenen Gründen liegt es nahe, bei knorpelchirurgischen Eingriffen so vorzugehen wie bei der unikompartimentellen Arthrose und die auslösende oder begleitende Achsabweichung mittels eines entlastenden Verfahrens zu behandeln. Ziel dieser Arbeit ist es, einen Überblick über nicht operative und operative Methoden der Druckentlastung eines Gelenkkompartiments bei knorpelchirurgischen Eingriffen (MFX, OATS, ACT) zu geben.

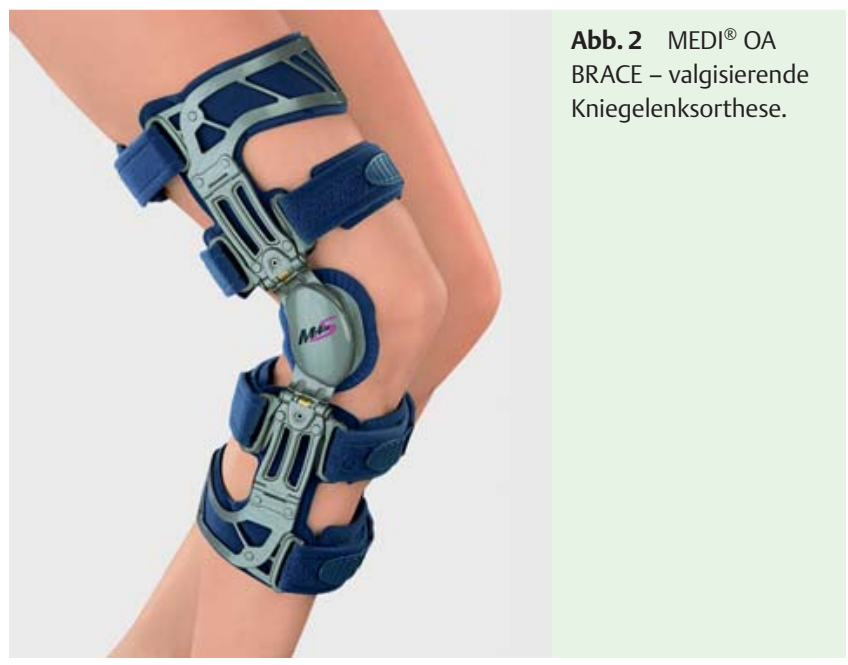

Nicht operative Entlastungsverfahren

$\nabla$

Zu den nicht operativen Verfahren zählen die Gewichtsreduktion, Schuheinlagen und Varus- und Valgusorthesen. Es ist in mehreren Studien belegt, dass Übergewicht einen bedeutenden Risikofaktor für das Neuauftreten von Knorpelschäden und einer initialen Kniegelenksarthrose darstellt [11-13]. Letztendlich sind die metabolischen Zusammenhänge neben der mechanischen Drucksteigerung aufgrund des Körpergewichts noch nicht abschließend verstanden. Inflammatorische Zusammenhänge scheinen bei Übergewichtigen die Entstehung eines Knorpelschadens zu begünstigen $[14,15]$. Diese Erkenntnisse legen nahe, dass eine Optimierung des Körpergewichts zum langfristigen Erfolg eines Knorpeleingriffs bzw. einer Entlastung anzustreben ist oder gar als Voraussetzung formuliert werden sollte.

Als weitere nicht operative Verfahren stehen Schuheinlagen und Orthesen zur Verfügung. Die Anwendung von keilförmigen Schuheinlagen ( $\bullet$ Abb. 1) und Kniegelenksorthesen ( $\bullet$ Abb. 2) sind bei der unikompartimentellen Kniegelenksarthrose weit verbreitet. Keilförmige Schuheinlagen auf der Gegenseite des ipsilateralen Knieschadens, also Keil lateral bei einem Knorpelschaden medial, können zwar das Adduktionsmoment sowohl in der Stand- und Abrollphase [16] wie auch beim Treppensteigen in geringem Maße reduzieren [17], ob Schuheinlagen temporär oder auch langfristig zu einer Entlastung eines Kompartiments sinnvoll sind, ist bislang nicht geklärt [18]. Der Effekt der Kompartimententlastung ist zudem begrenzt. So konnte eine durchschnittliche Druckreduktion bei einem $10 \mathrm{~mm}$ Keil von 2-7\% in vivo nachgewiesen werden [18].

Valgisierende Orthesen hingegen erzielen eine Entlastung des medialen Kniegelenkskompartiments in der Ganganalyse nicht nur während der Standphase, sondern auch während der Schwungphasen $[19,20]$. In vergleichenden Studien konnte gezeigt werden, dass bei medial betonter Gonarthrose $20 \%$ der behandelten Patienten von einer valgisierenden Kniegelenksorthese profitierten, wohingegen nur $13 \%$ der Betroffenen von einer Besserung mittels Schuheinlagen mit Außenranderhöhung berichteten [21]. Eine anatomische Untersuchung an 5 Leichenpräparaten konnte zeigen, dass durch die Verwendung einer entsprechenden Orthese eine Entlastung des medialen oder lateralen Kniegelenkskompartiments erreicht werden kann. Im Versuchsaufbau unter Kompression mit $1500 \mathrm{~N}$ ergab sich unter Anlage einer Varusknieentlastungsorthese eine prozentuale Entlastung 

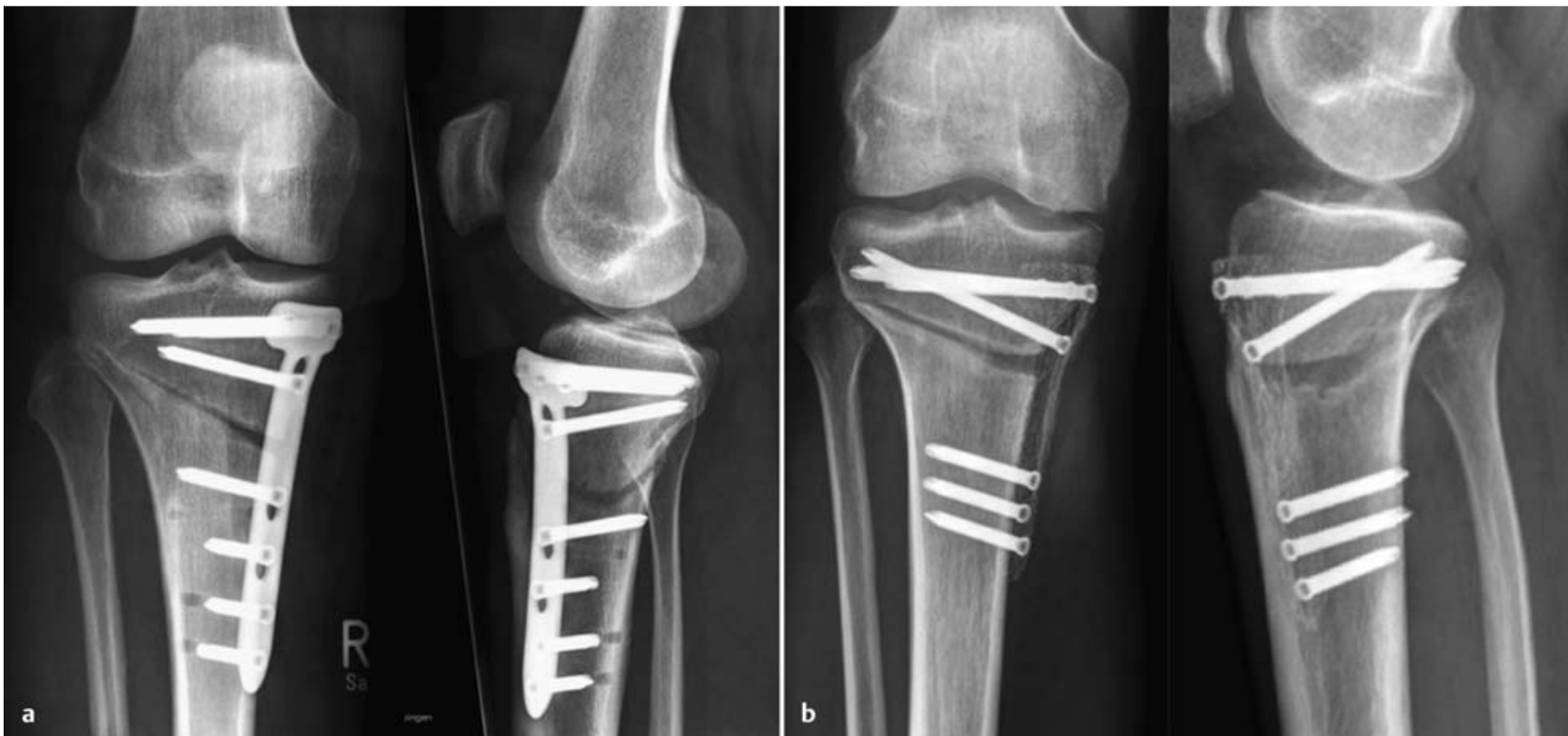

Abb. 3 a und b a Valgisierende Open-Wedge-HTO - mit TomoFix Platte (Synthes ${ }^{\circledR}$, Umkirch, Deutschland). b Valgisierende Open-Wedge-HTO - mit PEEK Power Plate (Arthrex ${ }^{\circledR}$, Karlsfeld, Deutschland).

des medialen Kompartiments von 70\% im Vergleich zur Messung ohne Orthese. Die Entlastung des lateralen Kompartiments unter Anlage einer Valgusknieentlastungsorthese betrug 46\% [22]. Mittels eines implantierbaren Druckmesssystems in die tibiale Komponente einer Knietotalendoprothese konnte die Arbeitsgruppe von Kutzner et al. diese Daten bestätigen [23]. Die Entlastung hing zudem von der Orthese ab. Bei $4^{\circ}$ bzw. $8^{\circ}$ valgus konnte eine Reduktion von $24 \%$ bzw. $30 \%$ des intraartikulären Druckes medial erreicht werden. Beim Treppensteigen hingegen reduzierte sich die Entlastung auf 24 bzw. 26\%. Generell ist jedoch bei den Betroffenen die Akzeptanz gering, dauerhaft eine valgisierende Kniegelenksorthese zu tragen [19].

\section{Operative „Unloading“-Verfahren: Umstellungsosteotomie und Druckentlastung $\nabla$}

Als operative Verfahren zur Entlastung von geschädigten medialen Gelenkabschnitten stehen dem Operateur mit dem arthroskopischen Release des Lig. obliquum posterior sowie der HTO (high tibial osteotomy) und DFO (distale femur osteotomy) etablierte Standardverfahren zur Verfügung [24,25]. Agneskirchner et al. [5] konnten zudem die Bedeutung des medialen Releases bei der Open-Wedge-HTO zur Druckentlastung des medialen Kniegelenkskompartiments zeigen. Eine 50\%ige Durchtrennung des medialen Kollateralbands senkte den Druck im medialen Kniegelenkskompartiment um $12 \%$. Die experimentelle, vollständige Durchtrennung des medialen Kollateralbands verringerte den Druck im medialen Kompartiment nach Open-Wedge-HTO gar um $24 \%$.

Während das arthroskopische Release des Lig. obliquum posterior eine Entlastung des medialen Kniegelenkskompartiments ohne eine Veränderung der Beinachse bewirken soll [26], beruht das Entlastungsprinzip der Open-Wedge-HTO auf der Verschiebung der Lastachse. Bei einer valgisierenden Open-Wedge-HTO oder valgisierenden Closed-Wedge-DFO erfolgt die Verschiebung der Lastachse nach lateral in das vermeintlich knorpelgesunde Kniegelenkskompartiment. Aus diesem Grund empfiehlt sich vor einer HTO oder auch einer DFO die arthroskopische Untersuchung des Kniegelenks [27]. Ferner können bei diesem Eingriff zusätzliche Schäden wie beispielsweise Meniskusläsionen therapiert werden $[28,29]$

Bei Patienten mit unklaren Erfolgsaussichten von einer Umstellungsosteotomie bzw. Zweifel des Patienten am Erfolg ist mittels „Brace-Test“ eine Simulierung der Entlastung möglich. Ein ganztägiges Tragen einer valgisierend/varisierenden Orthese für 6-8 Wochen kann eine nachfolgende Umstellungsosteotomie bereits präoperativ simulieren und somit Aufschluss über eine erwartbare Beschwerdeerleichterung geben [30]. In einer Studie von Minzlaff et al. wurde bei 29 Patienten eine valgisierende Orthese (Medi M4OA - s. $\odot$ Abb. 2) ganztags angepasst. Nach 6-8 Wochen wurden die Beschwerden erneut nach der VAS bestimmt. Dabei zeigte sich eine signifikante $(p<0,001)$ Schmerzreduktion von VAS7 (3-9) auf VAS2 (0-7) [30]. Im Folgenden wurden 14 Patienten mittels HTO versorgt; 11 Patienten konnten postoperativ erfasst werden. Bei 6 Patienten entsprach der VAS-Wert dem der Orthesenbehandlung, bei 4 Patienten war der VAS-Wert geringer und bei 1 Patienten höher.

Das Prinzip der Druckentlastung im betroffenen Kompartiment durch eine valgisierende Open-Wedge-HTO ( $\bullet$ Abb.3a) bzw. valgisierende Closed-Wedge-DFO oder durch eine varisierende Closed-Wedge-DFO oder varisierende Closed-Wedge-HTO ist eine anerkannte Methode bei medialer respektive lateraler Gonarthrose [31]. Essenziell ist eine detaillierte präoperative Planung, um die Deformität zu analysieren und die Korrektur gemäß der Deformität durchzuführen. Bei zeichnerischer Planung konnte in retrospektiven Untersuchungen eine Abweichung von $2,5^{\circ}$ $\pm 3,4^{\circ}$ im Sinne einer Unterkorrektur gezeigt werden [32]. Mit einer hohen Intra- und Interobserverreliabilität zwischen unterschiedlicher Software kann von einer zunehmenden Genauigkeit ausgegangen werden [33]. Bei Unterkorrektur mit unzureichender Druckentlastung im medialen Kompartiment kann der Erfolg 


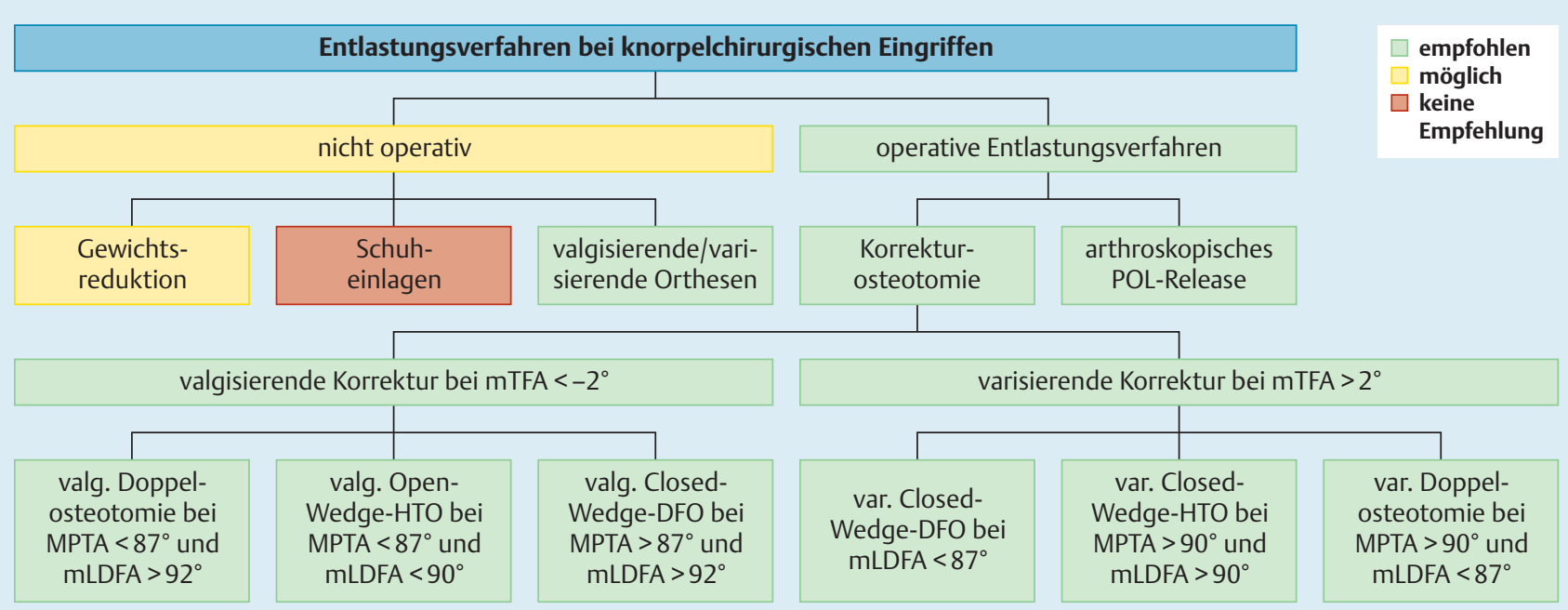

Abb. 4 Behandlungsalgorithmus zur Druckentlastung am Kniegelenk (mTFA: mechanical tibial-femoral angle; MTPA: medial proximal tibial angle; mLDFA: mechanical lateral distal femur angle).

der Open-Wedge-HTO reduziert sein. Nach knorpelreparativen Maßnahmen und Unterkorrektur der Open-Wedge-HTO kann zudem das gesamte Knorpelregenerat gefährdet sein.

In vielen klinischen Untersuchungen bei medialer Gonarthrose wurde eine im lateralen Kniegelenkskompartiment verlaufende Beinachse als ideales postoperatives Ergebnis beschrieben. Seit der Arbeit von Fujisawa 1979, die auf arthroskopischen Befunden von kleinen Gruppen beruht, wird vielerorts in der Behandlung der medialen Gonarthrose die postoperative Beinachse auf $62 \%$ (Tibiaplateaubreite, medial 0\%, lateral 100\%) geplant. Im Falle eines fokalen Knorpelschadens und Knorpelchirurgie ist bei begleitender HTO unserer Meinung nach die Verlegung der Beinachse über $60 \%$ nicht erforderlich. Wir streben intraoperativ als Schnittpunkt Tibiaplateau/mechanische Achse $55-60 \%$ bzw. ein mTFA von $1-2^{\circ}$ valgus an. Bei degenerativen Veränderungen sollte hingegen je nach Stadium ein Ziel von ca. $60-66 \%$ bzw. ein mTFA von $2-4^{\circ}$ erreicht werden [34]. Eine Korrektur mit einem MPTA (medialen proximalen Tibiawinkel) über $94^{\circ}$ sollte jedoch nicht resultieren. Bei entsprechenden Deformitäten kann daher auch eine Doppelosteotomie (Kombination aus Open-WedgeHTO und Closed-Wedge-DFO) zur ausreichenden Korrektur erforderlich werden. Studienergebnisse, die diese Ansicht bestätigen, stehen noch aus.

Nach erfolgreicher Anwendung im Tiermodell mit guten biomechanischen Eigenschaften [35] finden zunehmend Implantate aus PEEK (Polyetheretherketon) ihre Anwendung in der Orthopädie und Unfallchirurgie. Die Vorteile dieser Implantate sind die hohe Steifigkeit, die Winkelstabilität und die Röntgendurchlässigkeit und damit bei der HTO die röntgenologische Beurteilbarkeit des plattennahen Osteotomiespalts ( $\bigcirc$ Abb. $\mathbf{3 b}$ ). Zudem werden bei CT- und MRT-Diagnostik weniger Artefakte beschrieben. In den klinischen Anwendungen der 1. Generation der PEEK Power Plate (Arthrex, Karlsfeld, Deutschland) konnten jedoch die Erwartungen nicht bestätigt werden [36]. Im Vergleich zur TomoFix Platte (DePuySynthes, Umkirch, Deutschland) zeigte sich eine höhere Komplikationsrate.

\section{Kontraindikationen für die kniegelenknahe Umstellungsosteotomie $\nabla$}

Bei der Wahl des additiven Operationsverfahrens neben der Knorpelchirurgie sollten neben der Compliance Risikofaktoren wie Übergewicht, Zigarettenkonsum [37] sowie Defektgröße, Beinachse und Kniegelenksstabilität berücksichtigt werden. Bei Rauchern oder beleibten Patienten sollte im Falle einer OpenWedge-HTO das Einbringen einer zusätzliche Spongiosaplastik in den Osteotomiespalt erwogen werden [37]. Nach einer aktuellen Untersuchung von Kohn et al. spielt das Patientenalter hingegen eine untergeordnete Rolle [38]. Eine Retropatellararthrose oder vorderer patellarer Knieschmerz sollte bei additiver Beinachsenkorrektur Beachtung finden. Unserer Meinung nach stellt eine retropatellare Symptomatik keine Kontraindikation für eine additive Beinachsenkorrektur dar $[34,39]$.

\section{Schlussfolgerung \\ 7}

Bei fokalem Knorpelschaden ist neben der Indikationsstellung für ein bestimmtes knorpelchirurgisches Verfahren die Druckentlastung der Schlüssel zum langfristigen Erfolg. Das knorpelchirurgische und das entlastende Verfahren sollten individuell auf die vorliegende Pathologie und den einzelnen Patienten zugeschnitten sein ( $\bigcirc$ Abb.4) [40]. Die Druckentlastung im Kniegelenk kann langfristig nur durch eine operative Beinachsenkorrektur erfolgen. Schuhranderhöhungen bzw. Einlagen erreichen keine ausreichende Entlastung. Kniegelenksorthesen können dieses Ziel erreichen, haben allerdings eine geringe Patientenakzeptanz.

Interessenkonflikt: Nein 


\section{Literatur}

1 Sterett WI, Steadman JR, Huang MJ et al. Chondral resurfacing and high tibial osteotomy in the varus knee: survivorship analysis. Am J Sports Med 2010; 38: 1420-1424

2 Minzlaff P, Feucht MJ, Saier T et al. Osteochondral autologous transfer combined with valgus high tibial osteotomy: long-term results and survivorship analysis. Am J Sports Med 2013; 41: 2325-2332

3 Gomoll AH, Filardo G, de Girolamo L. Surgical treatment for early osteoarthritis. Part I: cartilage repair procedures. Knee Surg Sports Traumatol, Arthrosc 2012; 20: 450-466

4 Bode G, Schmal H, Pestka JM et al. A non-randomized controlled clinical trial on autologous chondrocyte implantation (ACI) in cartilage defects of the medial femoral condyle with or without high tibial osteotomy in patients with varus deformity of less than 5 degrees. Arch Orthop Trauma Surg 2013; 133: 43-49

5 Agneskirchner JD, Hurschler C, Wrann CD et al. The effects of valgus medial opening wedge high tibial osteotomy on articular cartilage pressure of the knee: a biomechanical study. Arthroscopy 2007; 23: 852-861

6 Mina C, Garrett WE jr., Pietrobon R et al. High tibial osteotomy for unloading osteochondral defects in the medial compartment of the knee. Am J Sports Med 2008; 36: 949-955

7 Halder A, Kutzner I, Graichen F et al. Influence of limb alignment on mediolateral loading in total knee replacement: in vivo measurements in five patients. J Bone Joint Surg Am 2012; 94: 1023-1029

8 Guettler JH, Demetropoulos CK, Yang KH et al. Osteochondral defects in the human knee: influence of defect size on cartilage rim stress and load redistribution to surrounding cartilage. Am J Sports Med 2004; 32: $1451-1458$

9 Guettler J, Glisson R, Stubbs A et al. The triad of varus malalignment, meniscectomy, and chondral damage: a biomechanical explanation for joint degeneration. Orthopedics 2007; 30: 558-566

10 Cicuttini F, Ding C, Wluka A et al. Association of cartilage defects with loss of knee cartilage in healthy, middle-age adults: a prospective study. Arthritis Rheum 2005; 52: 2033-2039

11 Brouwer GM, van Tol AW, Bergink AP et al. Association between valgus and varus alignment and the development and progression of radiographic osteoarthritis of the knee. Arthritis Rheum 2007; 56: 12041211

12 Felson DT. Obesity and vocational and avocational overload of the joint as risk factors for osteoarthritis. J Rheumatol Suppl 2004; 70: 2-5

$13 \mathrm{Niu}$ J, Zhang YQ Torner J et al. Is obesity a risk factor for progressive radiographic knee osteoarthritis? Arthritis Rheum 2009; 61: 329-335

14 Goldring $M B$, Otero M. Inflammation in osteoarthritis. Curr Opin Rheumatol 2011; 23: 471-478

15 Berenbaum F, Eymard F, Houard X. Osteoarthritis, inflammation and obesity. Curr Opin Rheumatol 2013; 25: 114-118

16 Shelburne KB, Torry MR, Steadman JR et al. Effects of foot orthoses and valgus bracing on the knee adduction moment and medial joint load during gait. Clin Biomech (Bristol, Avon) 2008; 23: 814-821

17 Alshawabka AZ, Liu A, Tyson SF et al. The use of a lateral wedge insole to reduce knee loading when ascending and descending stairs in medial knee osteoarthritis patients. Clin Biomech (Bristol, Avon) 2014; 29: 650-656

18 Kutzner I, Damm P, Heinlein B et al. The effect of laterally wedged shoes on the loading of the medial knee compartment-in vivo measurements with instrumented knee implants. J Orthop Res 2011; 29: 1910-1915

19 Brouwer RW, van Raaij TM, Verhaar JA et al. Brace treatment for osteoarthritis of the knee: a prospective randomized multi-centre trial. Osteoarthritis Cartilage 2006; 14: 777-783

20 Schmalz T, Blumentritt S, Drewitz H. Die Nutzung von Unterschenkelorthesen im Rahmen der konservativen Behandlung der Gonarthrose. Med Orth Tech 2011; 131: 68-78

21 van Raaij TM, Reijman M, Brouwer RW et al. Medial knee osteoarthritis treated by insoles or braces: a randomized trial. Clin Orthop Relat Res 2010; 468: 1926-1932
22 Donjoy OA, König DP, Güngör Ü et al. Orthese bei Gonarthrose - eine anatomische Studie. Meeting Abstract. Deutscher Kongress für Orthopädie und Unfallchirurgie, 70. Jahrestagung der Deutschen Gesellschaft für Unfallchirurgie, 92. Tagung der Deutschen Gesellschaft für Orthopädie und Orthopädische Chirurgie. Düsseldorf, Köln: German Medical Science; 2006: DocE.7.2.-1387

23 Kutzner I, Kuther S, Heinlein B et al. The effect of valgus braces on medial compartment load of the knee joint - in vivo load measurements in three subjects. J Biomech 2011; 44: 1354-1360

24 Lobenhoffer P, Agneskirchner JD. Improvements in surgical technique of valgus high tibial osteotomy. Knee Surg Sports Traumatol Arthrosc 2003; 11: $132-138$

25 Staubli AE, De Simoni C, Babst R et al. TomoFix: a new LCP-concept for open wedge osteotomy of the medial proximal tibia-early results in 92 cases. Injury 2003; 34 (Suppl. 2): B55-B62

26 Atoun E, Debbi R, Lubovsky O et al. Arthroscopic trans-portal deep medial collateral ligament pie-crusting release. Arthrosc Tech 2013; 2: e41-e43

27 Müller $M$, Strecker $W$. Arthroscopy prior to osteotomy around the knee? Arch Orthop Trauma Surg 2008; 128: 1217-1221

28 Niemeyer P, Koestler W, Kaehny C et al. Two-year results of open-wedge high tibial osteotomy with fixation by medial plate fixator for medial compartment arthritis with varus malalignment of the knee. Arthroscopy $2008 ; 24: 796-804$

29 Schröter S, Gonser CE, Konstantinidis L et al. High complication rate after biplanar open wedge high tibial osteotomy stabilized with a new spacer plate (Position HTO plate) without bone substitute. Arthroscopy 2011; 27: 644-652

30 Minzlaff P, Saier T, Brucker PU et al. Valgus bracing in symptomatic varus malalignment for testing the expectable "unloading effect" following valgus high tibial osteotomy. Knee Surg Sports Traumatol Arthrosc 2014; [Epub ahead of print]

31 Floerkemeier S, Staubli AE, Schröter $S$ et al. Outcome after high tibial open-wedge osteotomy: a retrospective evaluation of 533 patients. Knee Surg Sports Traumatol Arthrosc 2013; 21: 170-180

32 Schröter S, Günzel J, Freude T et al. [Precision in the planning of open wedge HTO.]. Z Orthop Unfall 2012; 150: 368-373

33 Schröter S, Ihle C, Mueller J et al. Digital planning of high tibial osteotomy. Interrater reliability by using two different software. Knee Surg Sports Traumatol Arthrosc 2013; 21: 189-196

34 Hinterwimmer S, Feucht MJ, Imhoff $A B$. Hohe tibiale Osteotomie bei Varusgonarthrose. Arthroskopie 2012; 25: 184-194

35 Rohner B, Wieling R, Magerl F et al. Performance of a composite flow moulded carbon fibre reinforced osteosynthesis plate. Vet Comp Orthop Traumatol 2005; 18: 175-182

36 Cotic $M$, Vogt $S$, Hinterwimmer $S$ et al. A matched-pair comparison of two different locking plates for valgus-producing medial open-wedge high tibial osteotomy: peek-carbon composite plate versus titanium plate. Knee Surg Sports Traumatol Arthrosc 2014; [Epub ahead of print]

37 Meidinger G, Imhoff AB, Paul J et al. May smokers and overweight patients be treated with a medial open-wedge HTO? Risk factors for non-union. Knee Surg Sports Traumatol Arthrosc 2011; 19: 333-339

38 Kohn L, Sauerschnig M, Iskansar S et al. Age does not influence the clinical outcome after high tibial osteotomy. Knee Surg Sports Traumatol Arthrosc 2013; 21: 146-151

39 Hinterwimmer S, Beitzel K, Paul J et al. Control of posterior tibial slope and patellar height in open-wedge valgus high tibial osteotomy. Am J Sports Med 2011; 39: 851-856

40 Feucht MJ, Minzlaff P, Saier T et al. Degree of axis correction in valgus high tibial osteotomy: proposal of an individualised approach. Int Orthop 2014; 38: 2273-2280 